\title{
A Public Ideation of Shape-Changing Applications
}

\author{
Miriam Sturdee, John Hardy, Nick Dunn, and Jason Alexander \\ Lancaster University, United Kingdom \\ $\{$ m.sturdee, j.hardy, nick.dunn, j.alexander\}@lancaster.ac.uk
}

\begin{abstract}
The shape-changing concept-where objects reconfigure their physical geometry-has the potential to transform our interactions with computing devices, displays and everyday artifacts. Their dynamic physicality capitalizes on our inherent tactile sense and facilitates object re-appropriation. Research both within and outside HCI continues to develop a diverse range of technological solutions and materials to enable shape-change. However, as an early-stage enabling technology, the community has yet to identify important applications and use-cases to fully exploit its value. To expose and document a range of applications for shape-change, we employed unstructured brainstorming within a public engagement study. A 74-participant brainstorming exercise with members of the public produced 336 individual ideas that were coded into 11 major themes: entertainment, augmented living, medical, tools \& utensils, research, architecture, infrastructure, industry, wearables, and education \& training. This work documents the methodology and resultant application ideas along with reflections on the approach for gathering application ideas to enable shape-changing interactive surfaces and objects.
\end{abstract}

\section{Author Keywords}

Shape-change; Shape-changing Interfaces; Shape-changing Applications; Brainstorming;

\section{ACM Classification Keywords}

H.5.2. Information Interfaces and Presentation (e.g. HCI): User Interfaces

\section{INTRODUCTION}

The study of shape-changing interfaces, displays, and objects is an emerging area of research. Shape-change allows objects to physically re-configure their external geometry to convey information [1], exploit perceived affordances in physical form [20], enhance output by exploiting the users' rich tactile sense [6], influence social behaviours [13], and re-appropriate objects through dynamic affordances [10].

Currently, shape-change research is driven by technology explorations or prototypes with a specific application focusdiverse user-led or applications-driven research has not yet

Permission to make digital or hard copies of all or part of this work for personal or classroom use is granted without fee provided that copies are not made or distributed for profit or commercial advantage and that copies bear this notice and the full citation on the first page. Copyrights for components of this work owned by others than ACM must be honored. Abstracting with credit is permitted. To copy otherwise, or republish, to post on servers or to redistribute to lists, requires prior specific permission and/or a fee. Request permissions from Permissions@ acm.org.

ITS '15, November 15-18, 2015, Funchal, Portugal.

Copyright (c) 2015 ACM. ISBN 978-1-4503-3899-8/15/11...\$15.00.

DOI: http://dx.doi.org/10.1145/2817721.2817734
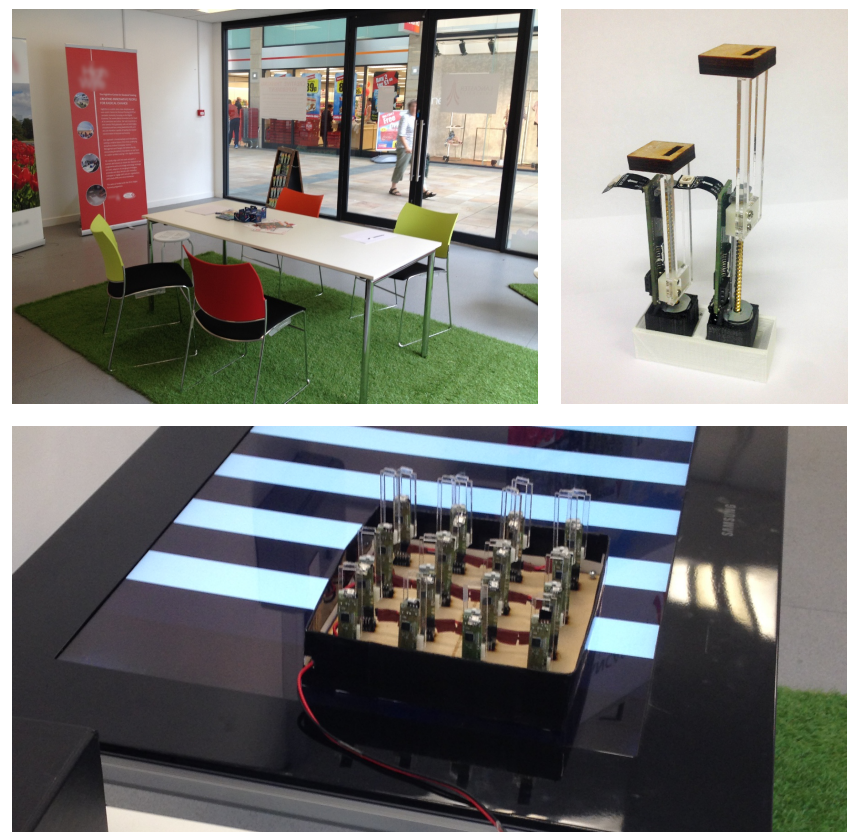

Figure 1. (Top left) Study location; (Top right) ShapeClip display components (minimum/maximum heights); (Bottom) Public demonstration using 16 ShapeClips [14].

occurred. Instead, we see a trend in the literature of documenting (for example): cartography $[1,28]$, wearable technology [3, 23], and mobile phone notifications [8, 12, 15]. However, to explore the full-potential and range of shapechange, and to focus technical development work, there is a need to better understand potential use-cases and applications [30] that drive future adoption.

This paper proposes a complementary way to address this knowledge gap through the analysis of 336 ideas generated by 74 members of the general public during a creative thinking experiment. We hypothesize that large public groups can ideate novel research directions and indicate requirements for shape change that are grounded in a diverse range of individual needs and demographics. Our findings include themes that expand and diversify the academic design space, and characteristics that help researchers reflect on producing appropriate solutions for the needs of a public user base. Specifically, over half of the generated ideas were categories such as Augmented Living, Entertainment, or Medical.

This paper aims to assist in generating future uses and research directions for shape-changing technology and subsequently contributes: (1) An unstructured brainstorming methodology involving non-expert individuals, carried out 
over seven days with 74 participants generating a total of 336 unique ideas; (2) Analysis of the generated ideas using Grounded Theory, identification of common theme categories, characteristics, and descriptive statistics; (3) Discussion around associated emergent themes, ideation output, and ideas relating to existing research (4) Reflection on the methodological approach-discussing generalisability, limitations, and considerations for future practitioners and; (5) A database of the generated ideas made available online at http://www.shape-change.org/brainstorm/.

\section{RELATED WORK}

\section{Existing Shape-changing Prototypes}

Shape-changing prototypes encompass a diverse range of materials, hardware, and usage scenarios. Many of these prototypes focus on a single application output (such as physically dynamic bar charts [36]) or interaction focus (displays that emulate reading a book [40]), or on material-based technological advancement of the field (Shape Memory Alloys [26] or partical jamming [32]), although there are cases where subsequent iterations of the same prototype have explored new application directions (e.g. inForm [10], deForm [9] and TRANSFORM [22]).

A large body of research in this area also looks at developing shape-changing versions of pre-existing technologies such as mobile phones [12, 15], tablets [35, 33] and desktops [37], although there are also more novel approaches considering artistic output [21] or emotive social-touch surfaces [24]. Another way in which research into these technologies progresses is to build upon previous prototypes incrementally, or to re-purpose components or ideas from existing work for development in other contexts. By following the citations within any given paper, justification for the prototype could be seen to come from the research community at large, rather than via ideation means.

\section{User-studies \& Prototype Evaluation}

Taking a user-centered approach for evaluation of a prototype is commonly seen in a commercial context although the details surrounding this methodology are not always given [18]. In academic research institutions it is common to ask colleagues/student participants to evaluate prototypes, or for studies to use low numbers of participants. Methodologies utilise observational studies either from product placement [13], or artistic installation [25]. Other issues surrounding participant selection due to local availability can stem from gender bias or incentivisation [41], and research familiarity [27]. This is not to say that researchers employing such methods of participant selection are not making valid contributions to the field, but that there is space for an expanded viewpoint around such studies.

\section{Brainstorming and Ideation}

Brainstorming is a methodology commonly employed within groups for freely generating ideas to solve a particular problem or to generally come up with new ideas. As a nonexperimental method, it is uncommon to see this kind of freeassociative thinking in scientific research. In contrast to the
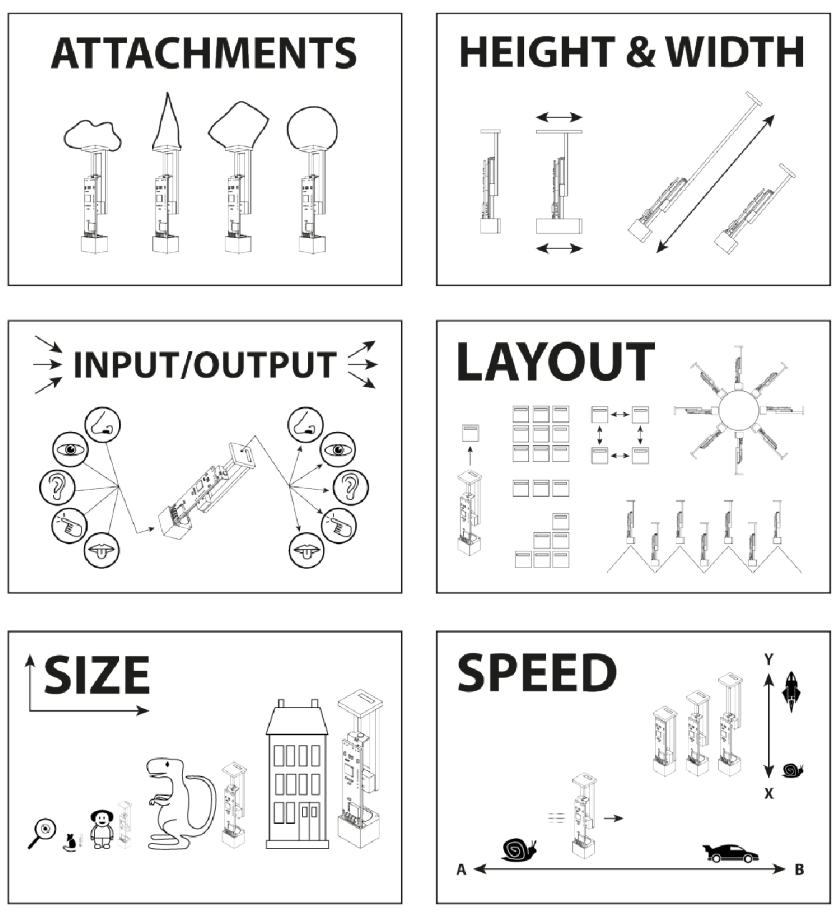

Figure 2. Indicative parameter posters

norm, Hardy et al's [14] experimental set-up utilised brainstorming to generate new research directions using designers and expanded upon these with rapid prototyping of viable ideas for shape-change. Notably, Jung et al. [17] also held sessions within their process (albeit with fewer participants). Utilising the general public in evaluation is unusual within the sphere of shape-changing interface research, although as previously mentioned both Gronvall et al. [13] and Nakajima et al. [25] successfully integrate a prototype within a public space. This allows both observation of diverse public interaction with shape-changing artefacts, and user testing in a nonpressurised setting. Follmer et al. hosted an open-house during which deFORM was showcased [9] but little information is provided as to demographic and experimental organisation. What is missing from research methodology in this area is purposeful and transparent recruitment of a participant pool from the general public. This paper hopes to elaborate upon participant selection and the use of non-institutional spaces in shape-changing research, following the success of such public-focused studies in co-design scenarios [31] and using brainstorming techniques such as De Bono's system outlined in Serious Creativity [7].

\section{METHODOLOGY}

The study goal was to generate shape-changing application ideas from a non-expert public group during an unstructured brainstorming session. These ideas were captured following demonstration of, and interaction with, an existing shapechanging display prototype. Analysis of the ideas isolated themes and characteristics of interest. By sampling a 'general public' user-base we hoped to: (1) obtain grounded application ideas that go beyond those currently documented; (2) ex- 
amine the effectiveness of public involvement; (3) compare and contrast ideas onto existing research literature.

\section{Experimental Setup and Location}

The study took place in a vacant retail unit with high footfall in a UK town-centre (Figure 1, top left). Banners invited the public to "Take part in a creative thinking experiment". No financial incentive/reward was offered. Participants self-selected, with minors required to be accompanied by an adult. Due to the random nature of such participation, data was collected individually, and without using published ideation techniques. This was to ensure consistency as no group facilitation or other structure could be planned for. The study ran over seven consecutive days including one weekend during school holidays. Alongside writing/drawing space, the unit contained a demonstration of ShapeClip prototyping units [14] and posters that facilitated the brainstorming/creative thinking task by communicating the theme of shape-change as a technology to the participants [11, 34]. These are detailed below and in Figures $1 \& 2$.

Shape-changing Display: In the study space was an example of a z-actuating shape-changing display using ShapeClip units [14] (Figure 1, top right). ShapeClips are modular prototyping tools containing individually programmable Arduino units. The grid demonstrated how vertical movement can be combined with visual output in a magic-lens style configuration [4]. Participants manipulated the ShapeClip lens by moving it across a Samsung SUR40 touch table over a variety of graphical outputs: checkerboard, stripes, sunburst gradient (Figure 1). Each individual shape-pixel actuated vertically between black/darkest output ( $0 \%$ actuation) to white/lightest output (100\% actuation) over a travel range of $60 \mathrm{~mm}$.

Parameter Posters: Six posters depicted possible shapechange parameters to consider: different attachments, height $\&$ width changes, different input/outputs, layouts, sizes, \& speeds. The posters served to broaden the range of divergent thinking by suggesting how the prototype might be altered (i.e. room-scale transformation) without overtly implying specific ideas, and regulated explanation of such parameters as part of the experimental design (Figure 2).

\section{Process}

The experimental process consisted of four stages: (1) Introduction \& Consent: The aims of the study were explained and appropriate consent forms completed. (2) Demonstration \& Interaction: The prototype was demonstrated, participants were encouraged to interact with the technology, and were shown parameter posters. (3) Ideation: Participants were asked-without time constraints, and on an individual basis - to generate as many uses for shape-changing technology as possible (previously generated ideas were not made available). Responses were paper-based rather than verbal, so as to capture ideas, and provide participants with a familiar medium with which they could express themselves without interference. Participants indicated when they had run out of ideas. (4) Exit Questionnaire: Participants were asked to provide demographic and other relevant written data as pertaining to the study.

\begin{tabular}{|c|c|c|}
\hline & Characteristic & Value \\
\hline 1 & Gender & Male (58\%), Female (42\%) \\
\hline 2 & Age (years) & $\mu: 30.07, \sigma: 14.10$, range $: 5-71$ \\
\hline 3 & Education & $\begin{array}{l}\text { School (12\%), } \begin{array}{c}\text { GCSE } \\
\text { Level/Vocational } \\
\text { (17\%), }\end{array} \text { Undergraduate } \\
\text { (34\%), Postgraduate } \\
(31 \%), \text { Ph.D }(3 \%)\end{array}$ \\
\hline 4 & Sector & $\begin{array}{l}\text { SciTech }(31 \%) \text {, Management \& Law }(10 \%) \text {, } \\
\text { Healthcare \& Medicine (4\%), Arts \& Social } \\
\text { Sciences (27\%), Unspecified (28\%) }\end{array}$ \\
\hline 5 & Creativity (1-5) & $\mu: 3.75, \sigma: 0.87$ \\
\hline 6 & $\begin{array}{l}\text { Tech Comfort } \\
(1-5)\end{array}$ & $\mu: 3.82, \sigma: 1.06$ \\
\hline 7 & Ownership & $\begin{array}{l}\text { Smartphone }(85 \%) \text {, Tablet }(64 \%) \text {, Laptop } \\
(84 \%) \text {, Desktop }(55 \%) \text {, Wearables }(9 \%) \text {, } \\
\text { Games Console }(62 \%)\end{array}$ \\
\hline 8 & Ideas Produced & $\begin{array}{l}\mu: 4.51, \sigma: 3.34, \text { range }: 1-21, q 1: 2, \\
q 3: 6\end{array}$ \\
\hline
\end{tabular}

Table 1. Descriptive characteristics of participants

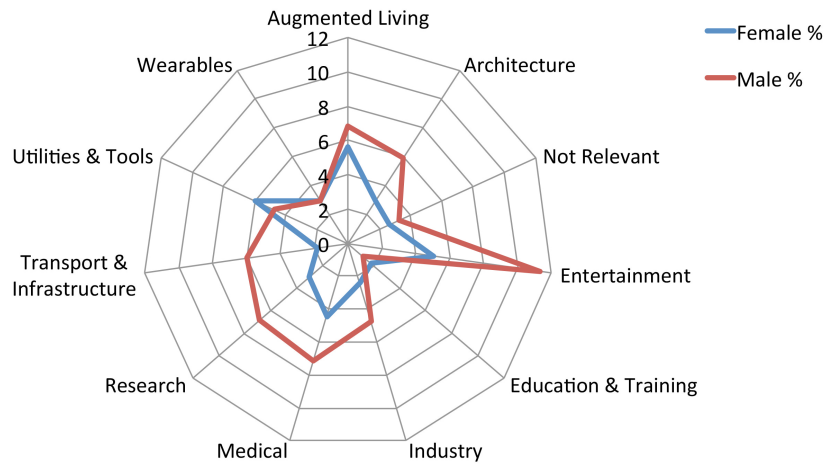

Figure 4. Percentage of ideas, per theme, by gender

\section{Data Analysis}

The demographic responses and raw ideas were collated and cross-referenced. All of the ideas were then coded according to the basic principles of Grounded Theory in three iterations. This resulted in clusters of idea themes, sub-themes, and characteristics (i.e. three independent 'idea feasibility' estimates), and a 3-point measure of elaboration. Ideas were also annotated with (approximate) scale, interactivity, and use of parameter posters. Our choice was informed by Rasmussen et al's classifications [30]. The data set was then queried by aspects of the questionnaire to identify any interactions between demography and participant output levels, i.e. age, gender, technological comfort, and also discover further variables that might improve the methodology.

\section{DATASET ANALYSIS}

\section{Participant Demographic}

Table 1 presents descriptive characteristics of the 74 sampled participants. The age ranged between 5-71 years ( $\mu: 30.07$, $\sigma: 14.10$, line 2 ). The majority held a university qualification $(67 \%$, line 3). On 5-point Lickert responses, people typically agreed with the statement that they were 'creative' $(\mu: 3.75$, line 5) and 'comfortable with technology' ( $\mu: 3.82$, line 6). The majority were technology consumers (line 7). Participants typically generated $(\mu: 4.51, \sigma: 3.34$, line 8$)$ ideas. 


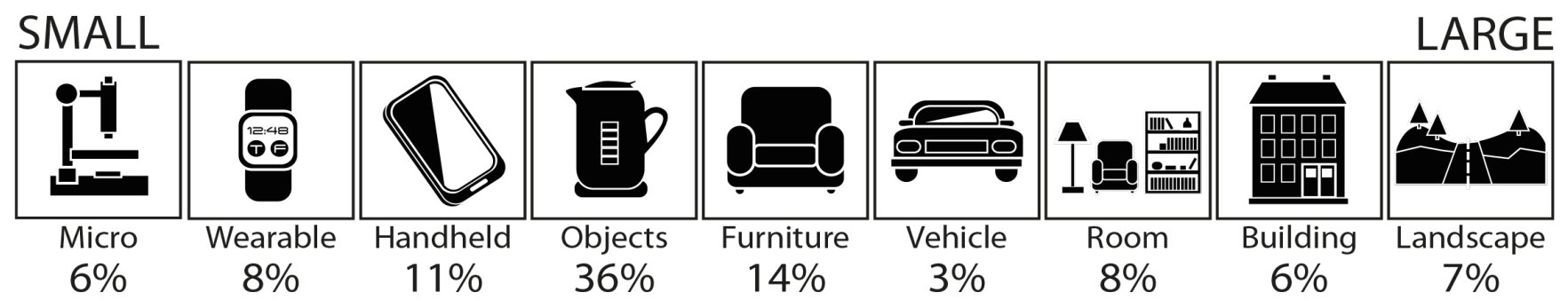

Figure 3. Ideas classified by approximate level of scale and type (Note: there is potential overlap between wearables/objects/hand-held but these were felt to be better represented as distinct entities for the purpose of scaling).

Systematic Chi-squared and correlation tests found no significant relationship between any of the demography characteristics and the number of ideas produced.

\section{Gender Differences}

An independent samples t-test was applied to the data to determine if there were significant gender differences between number of ideas generated. There was no significant difference in the number of ideas for men $(\mathrm{M}=4.767, \mathrm{SD}=2.843)$ and women $(\mathrm{M}=4.226, \mathrm{SD}=3.955) ; \mathrm{t}(0.686)=72, \mathrm{p}=0.495$ (there were no participants who identified with other gender identities). However, plotting the thematic data by gender does suggest that there may be evidence of bias toward particular themes (see Figure 4). A larger sample would be required for generalisation, but it does indicate the importance of mixed groups.

\section{Idea Themes}

A total of 336 ideas were generated. These ideas were presented using drawings (6\%), writing $(46 \%)$, and a mixture of both $(48 \%)$. Grounded coding sessions produced 11 major themes. The themes identified for using shape-change were (with examples for each) Entertainment (physical 3D television, drawings which come to life); Augmented Living (responsive fake plants, furniture that responds to the body); Medical (beds to reduce pressure on injuries, responsive prosthetic limbs); Utensils and Tools (re-sizable joinery tools, reactive camera tripod); Research (responsive sculpting materials, "Holodeck"); Architecture (earthquakeresponsive building foundations, reconfigurable rooms); Infrastructure (flood-resistant bridges, roads which respond to accidents); Industry (remote engineering in space, moulds for slip-casting); Wearables (re-sizable bags, anti-mugging wallet); and Education \& Training (3D white-boards, shapechanging museum exhibits), with $6.2 \%$ of ideas counting as 'not relevant' (headphones that play music when they sense your ear, voice controlled ovens).

Each theme also contained associated sub-categories and cross-over: i.e. a medical bed for coma patients might have a primary theme of Medical but a secondary theme of Augmented Living with a minor catergory of Furniture. The ideas with associated major, minor and secondary themes can be seen at http://www.shape-change.org/brainstorm/.

Table 2 summarises the total idea database, the associated categories, and lists these according to most frequent to least frequent. The most ideas were generated in the Entertainment category, with Education \& Training the least populated idea category. A category for ideas that did not map onto the theme of shape change, or were insufficiently explained was included here as this category comprises a portion of the data set on a par with Industry, or Wearables. It is not clear whether participants generating unrelated ideas were simply carried away by the brainstorming task, or misunderstood the parameters of the study.

Themes are described in more detail in the discussion, alongside sample ideas in Table 2. This also presents small graphs that indicate idea feasibility and elaboration within each theme, as well as showing idea frequency per theme.

Feasibility: Each idea was coded according to how viable it was to produce this item using current levels of technology, with a high value (5) indicating that it could be built almost immediately, and a low value (1) indicating only a slight probability that the item could be built, even in long time-frame. Average idea feasibility across the entire data set on a 5-point scale was $(\mu: 2.72, \sigma: 0.77)$ (scored from unfeasible at the lower end, to very feasible at the higher end). Coding was carried out independently by three researchers in the shape-change field using the same method, after which an average feasibility level was generated. Feasibility by theme can be seen in column 4 (Table 2), darker blue indicates higher feasibility, e.g. ideas within the Augmented Living and Entertainment themes appear to be more feasible given current technological advances than Infrastructure or Architecture - possibly because shape-change in the home/office is already in place, albeit at a lower level of technology (adjustable office chairs), but also at the research stage (shape-changing bench [13], or computer game controller [42]).

In comparison, architectural and landscape/infrastructure level shape-change would require not only a large amount of resource/space, but also significant changes in highly regulated building practices. Although there are real life examples (automatically raising road barriers, meetingroom dividers) in daily use, these are at a much smaller scale of what would be necessary to realise the generated ideas in these themes. Comparatively, the Wearables category shows a lower feasibility, possibly because the ideas generated rely on microscopic shape change (high shaperesolution) that is not currently in evidence in day to day 


\begin{tabular}{|c|c|c|c|c|}
\hline Theme & Description & Freq. & Feasibility & $\overline{\text { Elaboration }}$ \\
\hline Entertainment & $\begin{array}{l}\text { Relating to devices, toys, games and other recreational activities such as sports or events. } \\
\text { E.g. Skate park ramps; } 3 D \text { chess; Sensory feedback for video-games; Physical } 3 D T V \text {. }\end{array}$ & $16.36 \%$ & $\mu 3.24 \quad \sigma 0.86$ & $\mu 0.74 \quad \sigma 0.75$ \\
\hline Augmented Living & $\begin{array}{l}\text { Improving general life via home improvements and/or smaller aspects of interior archi- } \\
\text { tecture. E.g. Form sensing furniture; Ventilation controls; Reactive aesthetics. }\end{array}$ & $12.79 \%$ & $\mu 3.33 \sigma 0.89$ & $\mu 0.79 \sigma 0.74$ \\
\hline Medical & $\begin{array}{l}\text { Based within the medical or inclusive living field, for the benefit of both staff and pa- } \\
\text { tients. E.g. Wheelchair ramps; Surgical staff training; Braille displays/announcements. }\end{array}$ & $11.60 \%$ & $\mu 3.21 \sigma 0.65$ & $\mu 0.74 \quad \sigma 0.63$ \\
\hline Utensils \& Tools & $\begin{array}{l}\text { Activity or task orientated hand-held, tabletop or other movable objects which fulfill a } \\
\text { set purpose. E.g. Multi-use screwdriver head; Intuitive weapons; Reactive pizza pan. }\end{array}$ & $10.71 \%$ & $\mu 2.94 \quad \sigma 0.82$ & $\mu 0.88 \sigma 0.66$ \\
\hline Architecture & $\begin{array}{l}\text { Large scale building forms and major interior alterations. E.g. Seismic-reactive build- } \\
\text { ings; Architectural visualization tools; Re-configurable rooms. }\end{array}$ & $8.92 \%$ & $\mu 2.63 \sigma 0.89$ & $\mu 0.70 \quad \sigma 0.59$ \\
\hline Infrastructure & $\begin{array}{l}\text { Concerned with roads and pathways, vehicles, or town/city level adaptations. E.g. Dy- } \\
\text { namic speed-bumps; Weather-responsive vehicles; Intuitive livestock fencing. }\end{array}$ & $7.73 \%$ & $\mu 2.08 \sigma 0.68$ & $\mu 1.07 \quad \sigma 0.48$ \\
\hline Education \& Training & $\begin{array}{l}\text { Educational context, specific training tools, and imagery. E.g. Physically interactive } \\
\text { white boards; Data communication in museums; } 3 \text { D instructions. }\end{array}$ & $2.97 \%$ & $\mu 3.10, \sigma 0.73$ & $\mu 1.00, \sigma 0.81$ \\
\hline Not Relevant & $\begin{array}{l}\text { Did not fit into shape changing parameters, possibly due to misunderstanding the con- } \\
\text { cept, or generated as adjunct to shape change. }\end{array}$ & $6.25 \%$ & $\mu 1.48, \sigma 0.81$ & $\mu 0.52, \sigma 0.60$ \\
\hline
\end{tabular}

Table 2. Idea themes, descriptions, and examples. Theme frequency, breakdowns of feasibility and elaboration are shown for each theme. Feasibility (5-point, red=unfeasible/blue=very feasible). Elaboration (3-point, red=minimal/blue=specific).

living or cutting edge research. The overall feasibility for all themes (excluding those not relevant) is high, showing that participants were able to generate ideas within the realms of practicality, whilst also thinking speculatively.

Elaboration: Participant responses ranged from single words (e.g. games) to long essays or highly detailed images with accompanying texts. Text responses made use of bullet points, as well as descriptive prose, and whilst the variety of explanation is interesting, there do not appear to be any indicators for type of response in comparison to demographic data. Elaboration was coded in the same way as feasibility, on a three point scale indicating how much information about each idea was available, after initial analysis indicated the range of such information. Highly elaborate (and relevant) ideas suggest a greater understanding of the prototypical technology on display, as well as an analytic mindset.

\section{Idea Properties}

Beginning with the poster parameters, $23 \%$ of ideas involved attachments to the actuators (such as soft coverings or building foundations, $14 \%$ required different heights / widths, $72 \%$ used inputs/outputs that integrated the shape-change with other systems or senses i.e. light, sound, $21 \%$ required nonrectangular or grid layouts of multiple actuators, $92 \%$ differed in size from the prototype shown, and 19\% required significantly different actuation speeds. These differences are worth noting as the chosen prototype has comparable technical specifications to works in recent literature (closed length: $80 \mathrm{~mm}$, fully actuated length: $120 \mathrm{~mm}$, maximum actuation speed: $80 \mathrm{~mm} / \mathrm{s}$ ), particularly in terms of size and actuation travel. This makes the resulting ideas even more interesting (1) because participants were able to generalise beyond the object we showed them; and (2) because the literature might be missing categories of actuation/ideas.

To explore the differences in scale and use between our ideaset and the literature, ideas were classified according to dominant scale; ranging from microscopic to landscapes. The percentage of the total ideas for each scale are shown in Figure 3. The largest number of responses at any one scale was 'object' (36\%), followed by furniture (15\%) and handheld $(11 \%)$. This indicates people are willing to see shapechanging devices as 'human sized'. The number of furniturebased shape-change ideas indicates the integration of repurposability/customisation into what surrounds us rather than stand-alone monolithic devices will be an important design consideration in the future.

To analyse interactivity, ideas were mapped onto Rasmussen's modes of interaction [30]. The majority of the generated ideas reacted automatically to conditions or input (indirect, $44 \%$ ) or were hypothesized as needing to have direct input to produce actuation (direct, $37 \%$ ). No human interaction $(16 \%)$ and remote interaction $(3 \%)$ made up the remainder. After our interpretation and categorisation of the ideas, we were surprised by the number of ideas that suggest operation without human interaction.

\section{DISCUSSION}

In addition to statistical and descriptive analysis, other themes and comparisons emerged from the data and are discussed below. These range from dimensionality, display/device com- 


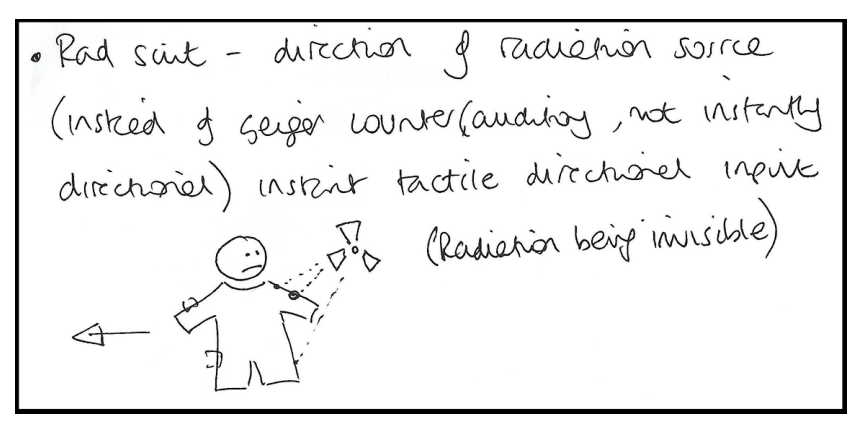

Simulating Nature using shape change electronicy

$\rightarrow$ Such as light!

$\rightarrow$ Use multiple shape change parts with plant components abtached to

simulale how plants respond to light aver ble day.

$\rightarrow$ Could be used nn a shopping cervere, such as bre frafford centre, to mane fake plentos more realistic

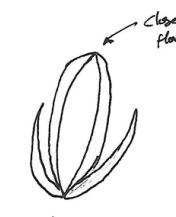
flower
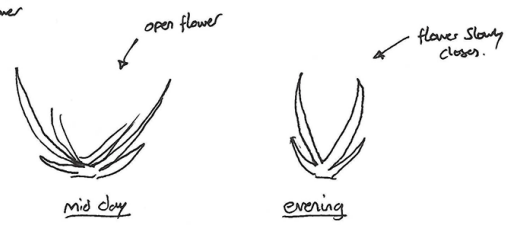

Figure 5. (Top) Reactive clothing; (Bottom) Shapechanging plants.

parison, validation of existing research from a public perspective, sustainable design, gaming, technology vs simplicity, and interdisciplinary thoughts around narrative imagery and temporality.

Most ideas do not exceed a single dimension of shapechange: Despite diverse applications and actuation scales within the idea-set, most ideas actuated to achieve a single purpose (e.g. unlocking a door) or transformation (e.g. moving 3D Braille paper) as opposed to multi-purpose shapechange. From our procedure it is difficult to know if there is no desire for 'generic objects', or if people could not ideate beyond simple actuation.

A dichotomy exists between displays and devices: The majority of ideas were devices (78\%) rather than displays (22\%). This differs from the focus of much technical research that looks at communication through dynamic visual display affordances. This indicates that initial shape-change applications may be welcome in day-to-day mundane scenarios. The main types of displays suggested were $3 \mathrm{D}$ televisions used for entertainment, or tangible browsers for online shopping.

Sense-making in shape-changing ideas: Participants were found to generate data that maps onto existing categories of shape-change and also makes sense to the world around them, such as realistic product predictions or feasible items. They tended not to ideate about things that could be enabled as the wider technological ecosystem evolves (for example, ideas which do not already link to existing items or structures). Ideas appear to be largely driven by desire (e.g. entertainment applications) or actual need (e.g. wheelchair adaptations) rather than technological speculation.

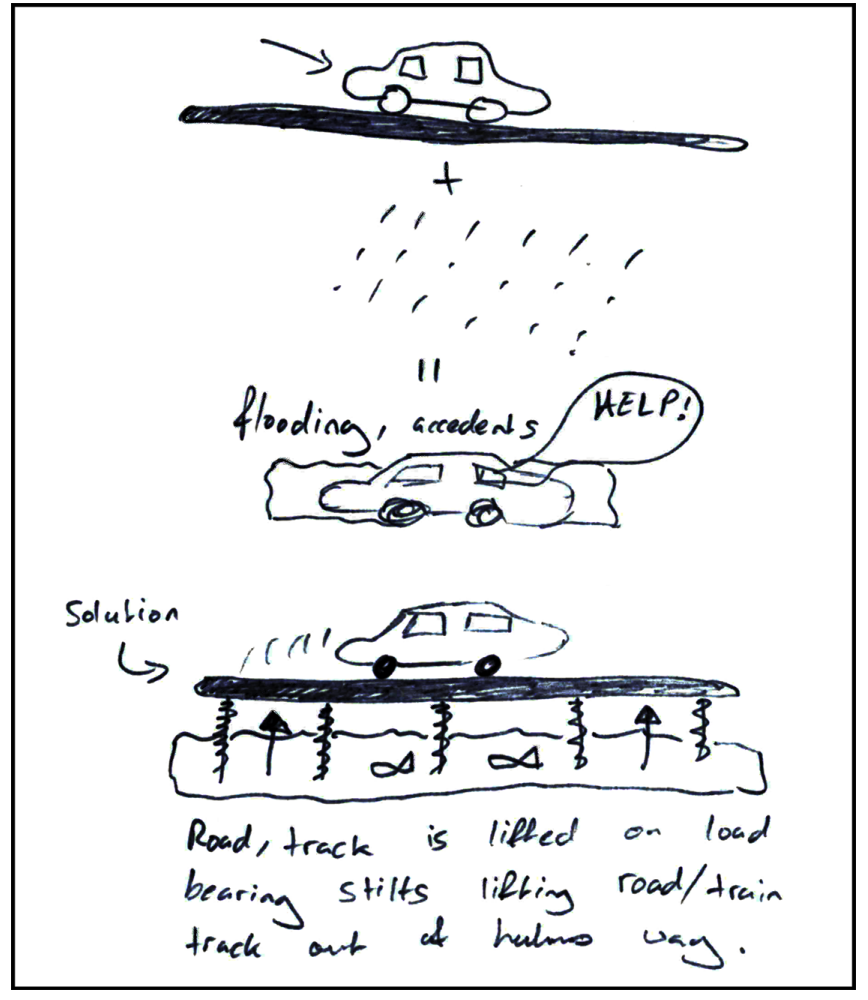

Figure 6. Narrative imagery describing shape-change

Familiar, mundane, self-actuated shape-change (e.g. convertible cars, automatic doors) did not play a major role in the idea-set. However, several ideas can be mapped onto existing prototypes, e.g. shape-changing vacuum cleaner head [39], shape changing coffee mugs [17], data visualisation [36], responsive plants [5] or air-quality reactive clothing [19].

This serves as validation for this method of consultation in two ways: (1) Current research projects are validated from a user-led perspective; and (2) Other ideas generated from the public data are thus likely to be viable directions for shapechanging application research.

The influence of nature: Rasmussen's review describes shape change being rooted in nature (the behaviour of the Southern White-faced Owl) [30]. This link was seen during the study duration whereupon participants either foresaw shape changing technology as attempting to purely emulate nature (responsive fake plants), using examples from nature to describe the movements they wished to outline (millipede walking platform) or create animal/technology forms with which to interact (interactive worm).

Use of narrative imagery to elucidate shape-change: The transitional nature of shape-changing technology lends itself to highly descriptive methods such as lengthy explanations or movements drawn in stages. This parallels comic strips in which advancement of time is shown over several drawings.

Several participants chose to explore their ideas using this methodology (see Figure 5), although there was a greater tendency for participants to draw one image and use text to explain the intricacies of their idea. This usage of narrative im- 
agery is already finding a place in shape change, be it in describing Ishii's futuristic Perfect Red [16] or Poupyrev et al's Lumen prototype [29]. Using narrative imagery to explore the nature of shape change is yet to be examined in research, yet appears to be vital in communicating complex iterations of these novel prototypes in research papers.

"Phygital" gaming: Entertainment was one of the most populated categories of idea, with gaming as a minor theme. Participants either suggested the notion of gaming in general, or leaned toward imagining highly specific versions of existing software such as Minecraft in 3D. Physical, non-console based gaming was also suggested - ideas in this sub-category ranged from chess pieces that reacted to illegal moves or cheating behaviours, to drawn imagery that fed into 3D playmats onto which toy cars could be placed, to Legos that maintained only a transient physical presence. This duality of ideas supports the phygital (physical/digital) presence of tangible user interfaces - occupying a space somewhere between the traditional table-top board game and richly detailed visual simulations or displays.

Overly technical solutions to simple problems: One potential issue in looking for applications for a novel technology, is that we may end up generating ideas that do not require actuated technology or a shape-changing display. This does not negate the ideas that were generated to specifically satisfy a desire rather than a need however (e.g. entertainment).

Such examples could be: a chair that adjusts to the shape of the bottom - already evident in memory foam; skinny jeans that adjust to the leg - achieved by stretchy materials such as lycra; or automatic ventilation systems - in use as airconditioners. Despite the existence of these products that do not relying on shape-changing gadgetry, this does not prevent novel technologies from eventually replacing their low-tech forerunners, such as micro-level shape-changing materials in clothing, which may then not only fit the form of the wearer, but also offer customisation opportunities.

Novelty of ideas between subjects: Despite the individual nature of the brainstorming methodology, in some cases, unrelated participants were seen to generate similar ideas over several categories. This duplication of ideas could be seen to indicate a collective desire for these technologies, or simply that these ideas are more obvious given the prompting of the prototype, posters and/or study environment. However, novelty within the dataset in some cases could also indicate ideas that are less feasible, or do not meet a practical need. To elaborate, below are some examples of duplication and novelty that were uncovered during analysis.

Examples of duplicated ideas: window blinds, re-sizable rooms, adjustable beds, prosthetic limbs, object detection for blind people, braille displays, real 3D TV, aerodynamic vehicle bodywork, re-sizable bags, flooding reactive bridges.

Examples of novel ideas: sun-shelters which grow when there is a high UV index, extendable target-sensing swords, shape-changing electric guitar, the perfect pizza-pan, Elizabethan suffocation collar, 3D police identi-fit, reactive shoe grips, interactive animal enclosures.

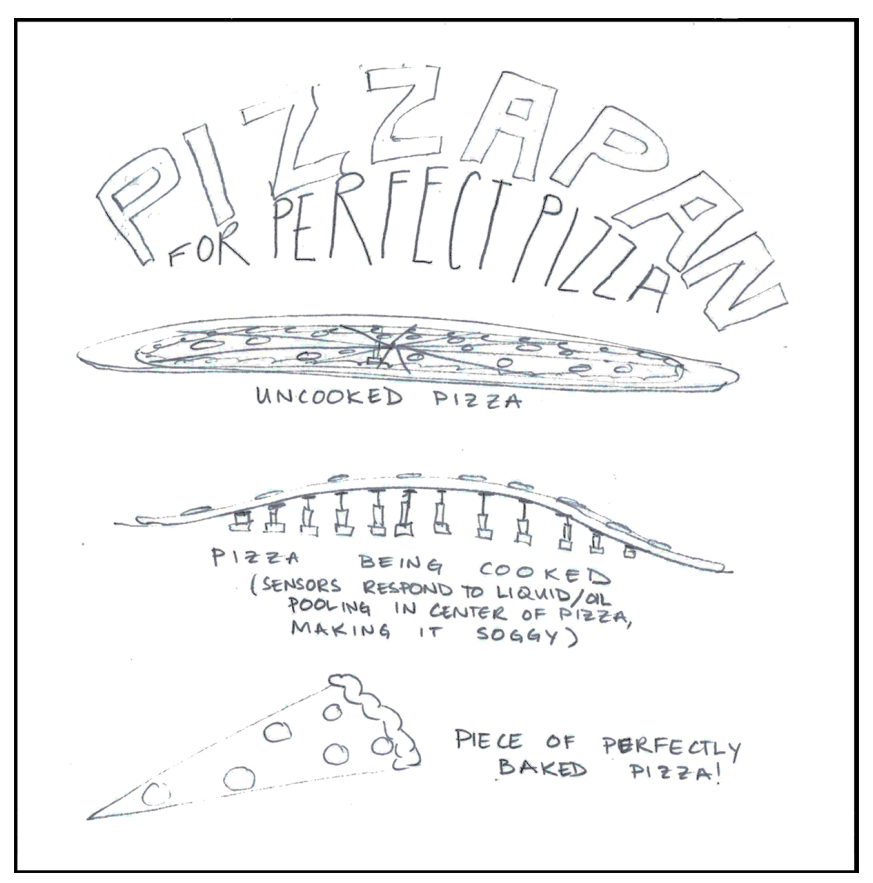

Figure 7. Novelty in idea generation - pizza-pan

Shape-change for sustainability: Themes surrounding sustainability are largely evident within the idea set, with several participants mentioning the housing crisis, or multiple/variable use objects (re-sizable pots, kettles (Figure 9), houses and aeroplanes). Successful commercialisation and implementation of shape-changing technology at levels from the micro to the macro could reduce drain on resources, overpopulation and lower waste production. Current prototypes which embrace both shape change and sustainability largely focus on design for behaviour change [38] rather than reducing the need for consumption in the first instance. An interdisciplinary approach working with designers in this field, might enable a valuable step forward in making these products a tangible reality.

The discussion above picks out the most interesting observations from the collected data but is by no means exhaustive. There may also be even more of interest from both the ideas themselves, and the qualitative data gathered post-ideation. What can also be taken away from this is that there is a wealth of information that can be gleaned from public engagement that is not only relevant to existing research, but can further inform shape-change, and other research.

\section{METHODOLOGICAL REFLECTION}

The experiment method was successful in collecting data on a volunteer basis from a general population without incentivising participation. The diversity of shape-changing applications generated is impressive, and, as we hoped, grounded in the needs and backgrounds of the sampled public. Having analysed the individual ideas, we are left with the impression that not only is there a demand for shape-changing technologies in mundane, day-to-day settings, but also that these technologies will be actively embraced. 

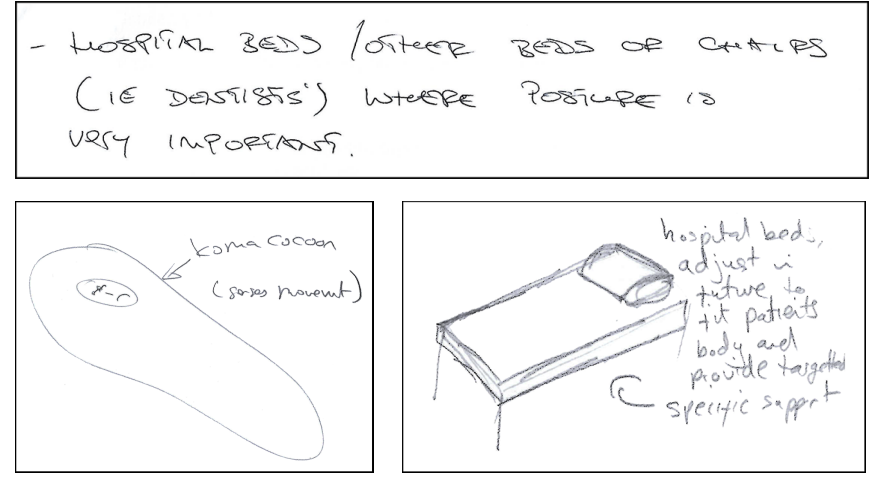

Figure 8. Duplication in idea generation

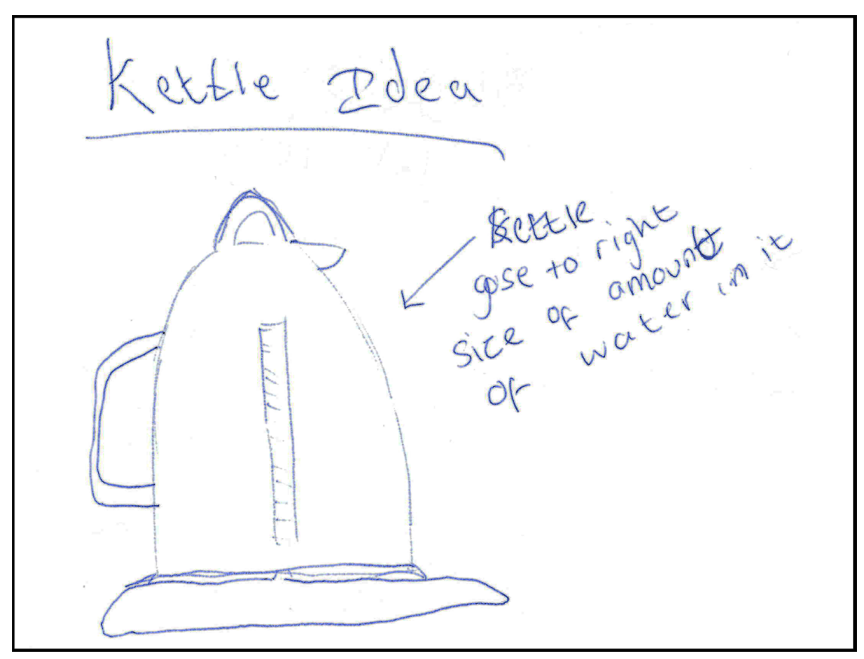

Figure 9. Adjustable, responsive kettle

\section{Interpersonal factors}

For the researcher(s) running the experiment, interpersonal factors were an important component of the delivery. Therefore any public-facing researcher must be able to engage on multiple levels, whilst delivering consistent communication and design in order to reduce the risk of bias. We specifically did not pressure any participants to engage, and felt this helped create a fruitful result. In some instances individuals entered the shop unit and asked to see the technology, but felt that they were not able to take part. This non-participatory interaction nevertheless suggests that there is a interest amongst the general public for engagement in new technologies.

\section{Prototype effects}

In terms of experimental limitations, the large number of object-level ideas is arguably linked to the object-level prototype. Although we took steps to expand this with the parameter posters, we suspect that if the study had been conducted with a material-level or room-sized prototype the results would have been different (although it is also possible that the ideas generated may have been more or less diverse) Shape-change is a very broad domain, and we hope that the characteristics of our idea-set will provide researchers with interesting reflections.

\section{Using public spaces for research}

Research using neutral non-laboratory spaces fulfills boundary principles [11] when used to run public facing studies, offers a possible solution to the skew that may be found when using an academia-specific participant pool/setting [2], and can produce a viable data set. Further work might investigate location-based differences as an adjunct.

Outside of the laboratory setting however, controlling all variables can present a difficulty. Despite this, the study is designed to be repeatable, and it can be seen that the specificity of the prototype used does not hinder the final data-set as participants were easily able to think outside of the study setting, and across themes/properties. Further work might focus on not only on varying location however, but also the study population, as identified subsets (such as designers or engineers) in the demographic could be isolated and then examined in more detail.

\section{Qualitative data}

Feedback questionnaires recorded demographic data and responses to the study design, demographic data did not contain any predictors relating to idea variation however. Comments relating to the prototype focused on seeing shape-change occur at a micro (i.e. material level), and wishing to experience variable actuation output in order to facilitate ideation.

Other types of qualitative data were also gathered which related to personal experience but did not offer an analytic viewpoint at this stage. Investigation of these types of responses may be beneficial however, as it could serve to enhance the human factor that can occasionally be missed from HCI research [2].

Some qualitative responses detailed the positive experience gained from being part of the research project, i.e. P34 "It is good to know that the public have had the opportunity to contribute ideas", P46 "Thanks for including me" and P40 "Interested to see where this tech goes". The overall response from the participant pool was overwhelmingly positive, supporting the possibility of applying this type of user study to other HCI research areas (e.g. wearables or mobile phones).

\section{Related methodologies}

Although this study is novel in its approach, it does bear some small similarity to Jung's SKIN methodology [17], in that brainstorming sessions were used, although Jung et al. used only 2 participants with art/design backgrounds to explore and draw concepts. What can be taken from this study's structure however, is the process with which ideas were developed and prototyped after the initial ideation process (see also [14]). The other study in which there is a parallel is Follmer et al's deFORM [9]) whose open-house style session invited a mixture of adults and children into the research setting to interact with the prototype, and informal feedback was given. In this case, however, no detail as to the background of the participants is given, and the focus of the open-house is not specific (i.e. were other prototypes on display?). This makes it difficult for other researchers to repeat the process. 
The ShapeClip brainstorming experiment [14] utilised the same base demonstration prototype as our study, but chose participants with a design-based background to generate ideas, and focused on developing these ideas in situ as part of a rapid prototyping experiment. There were also less ideas generated (86 compared to 336), via fewer participants, and across fewer themes, although more ideas were generated per participant. There were similarities within the themes however, such as Augumented Living and Wearables, although ideas mapped specifically onto the ShapeClip prototype, rather than shape-change in general. The difference in format (full day workshop as opposed to drop in session of self-selecting length) might have led to pressure to generate more ideas than the public study. Additionally, by using experienced designers, there is a tendency for individuals to have familiarity with such processes. With regards to these preceding research scenarios, our ideation study can be seen as not only responding to Banon's call [2], but also building on portions existing research.

\section{Reflection Summary}

This work shows that institutional public relations can be enhanced, a larger participant pool accessed, and useful product and theory-level ideas gathered for which there is a user-demand by utilising accessible public spaces in shapechanging research. This builds upon previous studies, and offers a viable alternative for research participant selection.

\section{FUTURE WORK}

Following the success of eliciting ideas in a non-research environment, it will be possible to further develop the methodology used here in an extended and revised fashion. Possible avenues of development might investigate whether using structured ideation techniques such as co-design might generate more cohesive results. Additionally, given that there are also differences for demographic factors (although nonsignificant at the current sample size), running specialised sessions for under $16 \mathrm{~s}$ or, for example, those with a background in the arts, might produce even more exciting data. There is also the possibility of using different shape-changing prototypes in the ideation sessions to explore differences in boundary objects/technology.

Work on the existing data set can also be taken a step further by developing several of the feasible ideas into working prototypes (as in Hardy 2015 [14]), or focusing existing research into areas that are desirable in a commercial setting [18]. Given that several of the ideas generated are already part of exciting research projects, it stands to reason that other ideas within the data set would produce meaningful results in the research setting and beyond.

\section{CONCLUSION}

In summary, 74 people generated 336 ideas that, after coding, split into 11 themes. These themes define directions for shape-change and insights into how and where people see it being used to solve problems in their day-to-day life. The responses of the public to the experiment were positive, and sufficient data was gathered to perform analysis and generate feasible ideas for future research directions.
The relative ease in which data was gathered suggests that an over-reliance on readily available participant pools is unnecessary, as an enthusiastic and diverse public can be surveyed if given the opportunity. Thus, the methodology implies that the current range of users for shape changing prototypes may be unnecessarily reliant on university-based data, which may produce bias. That is not to say that this data should be disregarded, but that it should be used comparatively with data collected from a wider pool.

Using an appropriate qualitative methodological approach it is possible to create a space in which a public participant pool can ideate around the theme of shape-change. The resulting data suggests that the public are not only able to generate ideas directly relating to current shape-changing research prototypes, but additionally, novel problem/desire-based directions for research. This reinforces previous calls for a more human-focused HCI [2] and shape-changing applications research perspective [30].

\section{ACKNOWLEDGEMENTS}

This research was supported by HighWire, a post-disciplinary Doctoral Training Centre at Lancaster University funded by the RCUK Digital Economy Programme through the EPSRC (Grant Reference EP/G037582/1), and partially-supported by GHOST, a project funded by the European Commission's 7th Framework Programme, FET-Open scheme (Grant \#309191).

\section{REFERENCES}

1. Alexander, J., Lucero, A., and Subramanian, S. Tilt Displays: Designing Display Surfaces with Multi-axis Tilting and Actuation. MobileHCI '12, ACM (2012), 161-170.

2. Bannon, L. J. Reimagining HCI: Toward a More Human-centered Perspective. Interactions 18, 4 (2011), 50-57.

3. Berzowska, J., and Coelho, M. Kukkia and Vilkas: Kinetic Electronic Garments. ISWC '05 (2005), 82-85.

4. Bier, E. A., Stone, M. C., Pier, K., Buxton, W., and DeRose, T. D. Toolglass and Magic Lenses: The See-through Interface. In SIGGRAPH '93, ACM (1993), 73-80.

5. Cheng, B., Gomes, A., Strohmeier, P., and Vertegaal, R. Mood fern: exploring shape transformations in reactive environments. ACE '14, ACM (2014), 60.

6. Cholewiak, R. W., and Collins, A. A. Sensory and Physiological Bases of Touch. The Psychology of Touch (1991), 23-60.

7. De Bono, E. Serious creativity. Journal for Quality and Participation (1995), 12-12.

8. Dimitriadis, P., and Alexander, J. Evaluating the Effectiveness of Physical Shape-change for In-pocket Mobile Device Notifications. CHI '14, ACM (2014), 2589-2592.

9. Follmer, S., Johnson, M., Adelson, E., and Ishii, H. deform: an interactive malleable surface for capturing $2.5 \mathrm{~d}$ arbitrary objects, tools and touch. UIST '11, ACM (2011), 527-536. 
10. Follmer, S., Leithinger, D., Olwal, A., Hogge, A., and Ishii, H. inFORM: Dynamic Physical Affordances and Constraints Through Shape and Object Actuation. UIST '13, ACM (2013), 417-426.

11. Fox, N. J. Boundary Objects, Social Meanings and the Success of New Technologies. Sociology 45, 1 (2011), $70-85$.

12. Gomes, A., Nesbitt, A., and Vertegaal, R. MorePhone: A Study of Actuated Shape Deformations for Flexible Thin-film Smartphone Notifications. CHI '13, ACM (2013), 583-592.

13. Grönvall, E., Kinch, S., Petersen, M. G., and Rasmussen, M. K. Causing Commotion with a Shape-changing Bench: Experiencing Shape-changing Interfaces in Use. CHI '14, ACM (2014), 2559-2568.

14. Hardy, J., Weichel, C., Taher, F., Vidler, J., and Alexander, J. Shapeclip: towards rapid prototyping with shape-changing displays for designers. $\mathrm{CHI}$ ' 15 , ACM (2015), 19-28.

15. Hemmert, F., Hamann, S., Löwe, M., Zeipelt, J., and Joost, G. Shape-changing Mobiles: Tapering in Two-dimensional Deformational Displays in Mobile Phones. CHI Extended Abstracts '10, ACM (2010), 3075-3080.

16. Ishii, H., Lakatos, D., Bonanni, L., and Labrune, J.-B. Radical atoms: beyond tangible bits, toward transformable materials. interactions 19, 1 (2012), $38-51$.

17. Jung, H., Altieri, Y. L., and Bardzell, J. Skin: designing aesthetic interactive surfaces. TEI '10, ACM (2010), $85-92$.

18. Kildal, J., Paasovaara, S., and Aaltonen, V. Kinetic device: designing interactions with a deformable mobile interface. CHI'12 Extended Abstracts, ACM (2012), 1871-1876.

19. Kim, S., Paulos, E., and Gross, M. D. Wearair: expressive $t$-shirts for air quality sensing. TEI ' 10, ACM (2010), 295-296.

20. Klemmer, S. R., Hartmann, B., and Takayama, L. How Bodies Matter: Five Themes for Interaction Design. DIS '06, ACM (2006), 140-149.

21. Kodama, S. Dynamic ferrofluid sculpture: organic shape-changing art forms. Communications of the ACM 51, 6 (2008), 79-81.

22. Leithinger, D., Follmer, S., Olwal, A., and Ishii, H. Physical telepresence: shape capture and display for embodied, computer-mediated remote collaboration. UIST '14, ACM (2014), 461-470.

23. Moere, A. V., and Hoinkis, M. A Wearable Folding Display for Self-expression. OZCHI '06, ACM (2006), 301-304.

24. Nakajima, K., Itoh, Y., Hayashi, Y., Ikeda, K., Fujita, K., and Onoye, T. Emoballoon. In Advances in Computer Entertainment. Springer, 2013, 182-197.

25. Nakajima, K., Itoh, Y., Yoshida, A., Takashima, K., Kitamura, Y., and Kishino, F. Fusa 2 touch display. SIGGRAPH '10, ACM (2010), 11.
26. Nojima, T., Ooide, Y., and Kawaguchi, H. Hairlytop interface: an interactive surface display comprised of hair-like soft actuators. WHC '13, IEEE (2013), 431-435.

27. Parkes, A., and Ishii, H. Bosu: a physical programmable design tool for transformability with soft mechanics. DIS '10, ACM (2010), 189-198.

28. Piper, B., Ratti, C., and Ishii, H. Illuminating Clay: A 3-D Tangible Interface for Landscape Analysis. CHI '02, ACM (2002), 355-362.

29. Poupyrev, I., Nashida, T., Maruyama, S., Rekimoto, J., and Yamaji, Y. Lumen: interactive visual and shape display for calm computing. SIGGRAPH '04 Emerging technologies, ACM (2004), 17.

30. Rasmussen, M. K., Pedersen, E. W., Petersen, M. G., and Hornbæk, K. Shape-changing Interfaces: A Review of the Design Space and Open Research Questions. CHI '12, ACM (2012), 735-744.

31. Sanders, E. B.-N., and Stappers, P. J. Co-creation and the new landscapes of design. Co-design 4, 1 (2008), 5-18.

32. Sato, T., Pardomuan, J., Matoba, Y., and Koike, H. Claytricsurface: An interactive deformable display with dynamic stiffness control. Computer Graphics and Applications, IEEE 34, 3 (2014), 59-67.

33. Schwesig, C., Poupyrev, I., and Mori, E. Gummi: a bendable computer. In CHI '04, ACM (2004), 263-270.

34. Star, S. L., and Griesemer, J. R. Institutional Ecology, Translations' and Boundary Objects: Amateurs and Professionals in Berkeley's Museum of Vertebrate Zoology, 1907-39. Social Studies of Science 19, 3 (1989), 387-420.

35. Steimle, J., Jordt, A., and Maes, P. Flexpad: highly flexible bending interactions for projected handheld displays. CHI '13, ACM (2013), 237-246.

36. Taher, F., Hardy, J., Karnik, A., Weichel, C., Jansen, Y., Hornbæk, K., and Alexander, J. Exploring interactions with physically dynamic bar charts. CHI '15, ACM (2015), 3237-3246.

37. Takashima, K., Aida, N., Yokoyama, H., and Kitamura, Y. Transformtable: a self-actuated shape-changing digital table. ITS '13, ACM (2013), 179-188.

38. Togler, J., Hemmert, F., and Wettach, R. Living interfaces: the thrifty faucet. TEI '09, ACM (2009), 43-44.

39. Vyas, D., Poelman, W., Nijholt, A., and De Bruijn, A. Smart material interfaces: a new form of physical interaction. CHI'12, ACM (2012), 1721-1726.

40. Watanabe, J.-i., Mochizuki, A., and Horry, Y. Bookisheet: bendable device for browsing content using the metaphor of leafing through the pages. UbiComp '08, ACM (2008), 360-369.

41. Weiss, M., Voelker, S., and Borchers, J. The benddesk demo: multi-touch on a curved display. ITS '10, ACM (2010), 317-317.

42. Ye, Z., and Khalid, H. Cobra: flexible displays for mobile gaming scenarios. CHI '10 Extended Abstracts, ACM (2010), 4363-4368. 